Administration, the single government payer, initiated My Health Bank program in 2014. The program is similar to Blue Button in US Medicare, in which each citizen can download personal medical claims data to personal computer.

Methods We developed ViMyHB smart phone app. Vi means visualisation and vitalization the content of My Health Bank. We will push customised personal relevant messages and reminds through ViMyHB app. If the users of ViMyHB app are satisfied with the information we provided, we will urge the user of to further provide other personal health related data to us, such as vital signs from wearable device or activities record in smart phone. We will value-added analyses these data and provide better information for better health promotion. In the third stage, we will develop some applications for injury prevention.

\section{REDUCING SECONDARY INJURY OF FARMERS WITH DISABILITIES WHEN USING TRACTORS: TECHNOLOGY}

Rebecca Brightwell. University of Georgia, USA

10.1136/injuryprev-2016-042156.1033

Background Farmers are very resilient and resourceful. After experiencing an injury that limits their mobility, they will often fabricate an adaptation that will enable them to return to farming. A review of homemade and commercial assistive technology products will be shown. The discussion will include the pros and cons of the different solutions in relation to re-injury and secondary injury.

Methods Through a project that assists farmers with disabilities and chronic health conditions, we have aided over 150 individuals to date with worksite accommodations and modifications. Once a farmer enters the program, a worksite assessment is completed that analyses tasks necessary to perform their job. After reviewing how the tasks are currently being performed, recommendations are made for safer and more effective alternatives that often involve adaptive equipment.

Results Farmers, when faced with disabilities and chronic health conditions, are very resourceful and will find creative ways to complete their tasks. However, many of these solutions may not be the safest alternative and often commercial solutions are more effective. The majority of the farmers in the project were unaware of technologies available to them. Implementation of the recommended solutions indicated a reduction of re-injury and the occurrence of secondary injuries.

Conclusions Farmers with disabilities and service professionals need to be aware of the various assistive technologies available. By using proper adaptive equipment and implementing worksite modifications, farmers can safely do many of the tasks they did before their illness or injury.

\section{Other Topics}

\section{Post Wed 3.13}

\section{LEVEL OF DISABILITY AMONG ADULT INJURY PATIENTS IN KENYA}

'Abdulgafoor M Bachani, 'Yuen Wai Hung, ${ }^{2}$ Stephanie Aketch, ${ }^{2}$ Ryan Duly, ${ }^{1}$ Kent A Stevens, 'Adnan A Hyder. 'Johns Hopkins International Injury Research Unit, Johns Hopkins University Bloomberg School of Public Health, USA; ${ }^{2}$ Handicap International, Kenya

10.1136/injuryprev-2016-042156.1034
Background Low- and middle-income countries have a disproportionally high burden of injuries. However, the short and longterm consequences of injuries on individuals remain largely unknown. While studies have found injuries among the top three causes of death among the urban poor in Kenya, little is known about the aftermath of injury among survivors in this largely young population.

Methods The Health, Economic and Long-term Social Impact of Injuries (HEALS) Study is currently being conducted in Kenya. Adult patients at least 18 years old who are hospitalised due to injury for one day or more in Kenyatta National Hospital are recruited to the longitudinal study. Baseline interview is conducted in the hospital, and follow-up interviews, at 1, 2, 4, and 12 month after hospital discharge, are completed via phone. Baseline interview includes questions about the injury that resulted in hospitalisation, associated costs and support received. Disability is measured using WHO Disability Assessment Schedule-II (WHODAS-II) at baseline and each subsequent follow-up interview.

Results Preliminary findings of the 320 respondents who completed the baseline interview show that majority $(92.8 \%)$ reported having no difficulty on all 6 domains of functioning prior to their injury (mean: 0.23 , SD: 1.19). At 1 month after hospital discharge, only $4.9 \%$ respondents $(n=247)$ reported having no difficulty in all domains of functioning (mean: 14.39, SD: 15.97). Exploratory factor analysis will be conducted to determine if the disability measure correlate with latent general disability in this population. Distribution of the disability score will be examined by severity of injury and types of injury.

Conclusions Preliminary findings suggest high level of disability at one month after hospital discharge. Data on subsequent follow-ups will strengthen the findings on short- and long-term burden of injuries in Kenya.

\section{EVALUATION OF A RISK SCREENING TOOL FOR COMPENSATION SYSTEM RECOVERY MANAGEMENT}

1,2Maatje Scheepers-Joynt, 'Dianne Sheppard, ${ }^{3}$ Meaghan O'Donnell, 'Sharon Newnam. ${ }^{1}$ Monash Injury Research Institute, Monash University, Australia; ${ }^{2}$ School of Health Sciences, Monash South Africa; ${ }^{3}$ Australian Centre for Posttraumatic Mental Health, Melbourne University, Australia

\subsection{6/injuryprev-2016-042156.1035}

Background Transport accidents result in Mental Health, pain and return-to-work (RTW) difficulties. To screen for risk of future poor outcomes assists early identification and effective recovery management for compensation systems that provide compensation for health care services post-accident. The purpose of this study was to evaluate the measurement properties of a predictive risk screening tool known as the Client Conversational Tool-Revised (CCT-R) within the recovery branch of the Transport Accident Commission (TAC), Victoria, Australia.

Methods De-identified data from 630 TAC clients who claimed compensation between April 2012 and October 2014 was analysed. Claims and payments data of later health service use was linked with associated CCT-R 'risk scores' for mental health, pain and RTW. Chronbach's alpha and Guttman Split-Half reliability analysis was used to evaluate internal consistency. Validity was assessed with analysis of receiver operating characteristice curves to estimate the area under the curve (AUC) for each CCT-R domain.

Results The mental health domain performed adequately with good reliability (Chronbach $\alpha=0.841$ ) and reasonable validity 
$($ AUC $=0.716 ;$ specificity, 76.84\%; sensitivity, 66.28\%). Poor validity evidence was observed for pain (AUC $=0.608$; sensitivity, 37.93\%; specificity, 83.74\%) and RTW domains (AUC $=0.534$; sensitivity, $18.99 \%$; specificity, 88.56\%). The RTW domain presented with good reliability (Chronbach $\alpha=0.847)$. The Pain domain could not be described as reliable (Chronbach $\alpha=0.039$ ).

Conclusions As a risk screening tool the CCT-R currently presents with discernible deficiencies relating to its measurement properties in two of the three domains. To facilitate early intervention decision management, CCT-R mental health scores could be useful, while pain and RTW domains require further revision.

\section{IMPROVING ESTIMATES OF THE COSTS OF INJURY IN WESTERN AUSTARLIA USING LINKED DATA}

'Delia Hendrie, ${ }^{1,2}$ Ted Miller, ${ }^{1}$ Sean Randall, ${ }^{1}$ Kate Brameld. ${ }^{1}$ Centre for Population Health Research, Curtin University Perth; ${ }^{2}$ Pacific Institute for Research and Evaluation, Maryland US

\subsection{6/injuryprev-2016-042156.1036}

Background Previous estimates of the costs of injury in Australia have relied heavily on secondary data, including from international sources. The availability of linked health data and other relevant datasets such as police reports of road crashes and personal injury motor vehicle insurance claims data has provided the opportunity to produce Australian-based estimates of the cost of injury using improved methods.

Methods A retrospective, population-based design was adopted in the study. Health data including emergency department presentations, hospital admissions and death data were linked with police crash report records and motor vehicle personal injury claims data by the Data Linkage Branch at the West Australian Health Department for 2002 through to 2014. Episodes of injury were determined using the linked health data, with clear zones established to account for return visits for the same injury. Corresponding injury costs were estimated based on generalised linear modelling of component costs of the injury claims data, with quality of life loss calculated from Global Burden of Disease disability weights.

Results Problems encountered in regards to the identification of episodes of injury will be discussed, in particular in regard to establishing clear zones. Similarly, issue in modelling from injury claims data for road injury casualties to all injury case will be highlighted. Previous estimates of the costs of injury will be compared with estimates based on the linked data approach and difference explored. Trends in injury costs in Western Australia over the past 10 -years will be analysed by socio-demographic and injury characteristics.

Conclusions Injuries are known to impose a significant health and social burden on individuals, families and the community. Applying improved methods of estimating these costs will provide the basis for better policy, planning and targeting of injury prevention strategies.

\section{REGIONAL DIFFERENCES IN SAFETY BEHAVIOUR AND ENVIRONMENTAL SAFETY, FINLAND 2013-2015}

Satu Helakorpi, Oona Pentala, Timo Koskela, Jukka Murto. National Institute for Health and Welfare (THL), Finland

10.1136/injuryprev-2016-042156.1037
Background Local authorities need a health and well-being monitoring system, that gives information on current safety behaviour and environmental safety and changes in them.

Methods The study is based on a nationally and regionally representative cross-sectional questionnaire (postal or web) survey entitled "The Regional Health and Well-being Study" (ATH) carried out in 2013-2015 in Finland. Respondents aged 20 years or more ( $n=89779$ ) were included. The indicators used were: 1 ) use of helmet always when cycling, and 2) slippery footpaths in winter near of respondents' home bother the respondents. Prevalence estimates with $95 \%$ confidence intervals were calculated. The results were presented by different population groups.

Results There were considerable differences in safety behaviour between educational groups, and they were most often unfavourable for the less-educated groups. These differences could generally be observed also at regional level. Use of helmet when cycling was most common in the highest educational group among both men and women. In the region of Uusimaa about $35 \%$ of cyclists reported always wearing a helmet when cycling whereas in Western Finland the corresponding prevalence was about $15 \%$. Slippery footpaths in winter were most common in the region of Uusimaa where over half of respondents reported that slippery footbaths bothered them, compared to $40 \%$ in Central and South-Eastern Finland.

Conclusions There were regional differences in both safety behaviour and environmental safety. In safety behaviour there were also sociodemographic differences. The ATH -study provides local authorities with the necessary tools to monitor their residents' health and well-being together with the factors affecting them. It is also an important tool for the monitoring of safety behaviour and environmental safety and their long- and shortterm changes in the adult population. The results are reported in an interactive online service (in Finnish): www.thl.fi/ath.

\section{FACTORS ASSOCIATED WITH MAXILLOFACIAL FRACTURES IN HASSAN DISTRICT, SOUTH INDIA: A RETROSPECTIVE STUDY}

${ }^{1}$ Utkarsha Lokesh, ${ }^{2}$ Shishir Kamath. 'Rajivgandhi Health University, Karnataka, India; ${ }^{2}$ Rajivgandhi Health University, Karnataka, India

\subsection{6/injuryprev-2016-042156.1038}

Background Injuries are the major public health problem in India. The present study was conducted to assess the aetiology, prevalence and pattern of facial fractures amongst the treated cases of maxillofacial fractures at the trauma centres in Hassan District, South India.

Methods Retrospective data of three years, 2011to 2014 was collected from records of five trauma-treating centres of a Hassan District in Southern India. Age, sex, aetiology, location of fractures and various other variables were collected.

Results Total 208 patients were treated in the hospitals for facial bone fractures. Men were more affected than women with a male to female ratio of $6: 1$. Nearly $50 \%$ of patients were aged between 21-30 years and road traffic injuries $(62.85 \%)$ caused most of the fractures, mostly among non-helmet two wheeler users. Road traffic injuries causing facial fractures occurred predominantly on weekdays (Tuesday and Wednesday). Chi-square test was used to test for significance and a $\mathrm{p}$-value $<0.05$ is regarded as significant. $60.35 \%$ of patients had fractures of mandible followed by $24.85 \%$ of zygomatic bone and $5.31 \%$ of maxillary bone. 